Anja K. Hartmann
Zwischen Differenzierung und Integration 
Forschung

Politikwissenschaft

\author{
Band 138
}


Anja K. Hartmann

\section{Zwischen Differenzierung und Integration}

Die Entwicklung des Gesundheitssystems in den Niederlanden und der Bundesrepublik Deutschland

Springer Fachmedien Wiesbaden GmbH 2002 
Inauguraldissertation zur Erlangung des akademischen Grades eines Doktors der Sozialwissenschaft der Ruhr-Univerität Bochum. Fakultät für Sozialwissenschaft 2000.

,Gedruckt auf säurefreiem und alterungsbeständigem Papier.

Die Deutsche Bibliothek - CIP-Einheitsaufnahme

\section{ISBN 978-3-663-11926-5 ISBN 978-3-663-11925-8 (eBook)}

DOI 10.1007/978-3-663-11925-8

\section{(C) 2002 Springer Fachmedien Wiesbaden}

Ursprünglich erschienen bei Leske + Budrich, Opladen 2002

Das Werk einschließlich aller seiner Teile ist urheberrechtlich geschützt. Jede Verwertung außerhalb der engen Grenzen des Urheberrechtsgesetzes ist ohne Zustimmung des Verlages unzulässig und strafbar. Das gilt insbesondere für Vervielfältigungen, Übersetzungen, Mikroverfilmungen und die Einspeicherung und Verarbeitung in elektronischen Systemen.

Einbandgestaltung: disegno, Wuppertal 


\section{Inhalt}

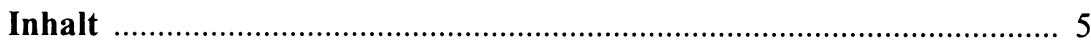

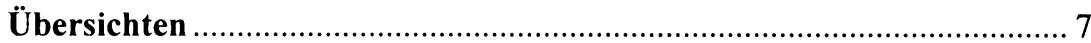

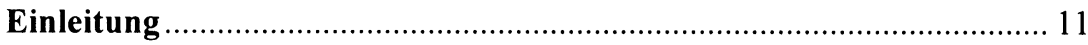

1 Differenzierung und Integration im Gesundheitssystem .................. 21

1.1 Gesundheit als ausdifferenziertes gesellschaftliches Teilsystem........21

1.1.1 Zum Begriff der Differenzierung .............................................21

1.1.2 Gesundheit unter systemtheoretischer Perspektive ..................24

1.1.3 Gesundheit unter akteurtheoretischer Perspektive ..................31

1.2 Gesundheit unter integrationstheoretischen Aspekten.........................37

1.2.1 ,Von der Medikalisierung zur Vergesellschaftung des Gesundheitssystems' .............................................................. 38

1.2.2 Die Verselbständigung gesellschaftlicher Teilsysteme ............ 46

1.2.3 Integrationsmechanismen: Interpenetration und Entdifferenzierung, (Selbst-) Steuerung und Reflexion ........... 54

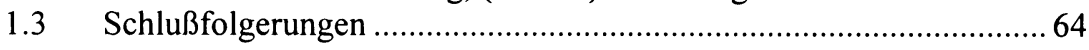

2 Bestandsanalyse des niederländischen und deutschen

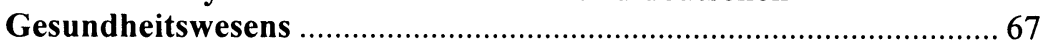

2.1 Gestaltungsmerkmale des niederländischen und deutschen

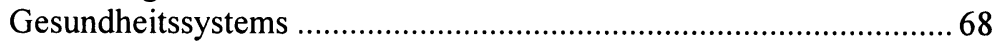

2.1.1 Gesundheit als korporatistisches Verbandswesen ....................68

2.1.2 Aufbau des Versorgungssektors............................................. 71

2.1.3 Aufbau des Versicherungs- und Vergütungssystems ...............75

2.2 Quantitative Ergänzung: Einige Daten zum niederländischen und

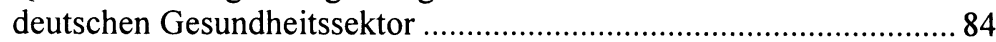

2.3 Schlußfolgerungen ............................................................... 90

3 Das Gesundheitssystem im Wandel der Wohlfahrtsgesellschaft ....... 93

3.1 ,Worlds of Welfare': Das deutsche und niederländische Gesundheitswesen im wohlfahrtlichen Kontext ..................................93

3.2 Statik und Dynamik wohlfahrtlicher Regimes................................... 103 
3.2.1 Phase 1 - Die Entstehung des Wohlfahrtsstaates................. 106

3.2.2 Phase 2 - Die Rekonstitution des Wohlfahrtsstaates ........... 112

3.2.3 Phase 3-Die Restrukturierung des Wohlfahrtsstaates........ 115

$3.3 \quad$ Schlußfolgerungen ............................................................. 123

4 Gesundheitspolitik im Vergleich: Streifzug durch die

Reformgeschichte........................................................................ 127

4.1 Gesundheitspolitik zwischen Selbststeuerung und Regulierung...... 127

4.2 Gesundheitspolitische Reformen im nationalen Vergleich............... 133

4.2.1 Reformpolitik in den Niederlanden: Von Hendriks zur ,managed liberalisation' ........................................................ 133

4.2.2 Reformpolitik in der BRD: Von Blanke zur Gesundheitsreform 2000 ................................................. 147

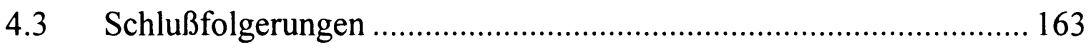

5 Modernisierungsstrategien in der Gesundheitsversorgung ............ 167

5.1 Die Folgen des ,Wohlfahrtspluralismus '....................................... 168

5.2 Modernisierungsstrategien im Versorgungssektor ........................ 173

5.2.1 Kooperation und Vernetzung ......................................... 173

5.2.2 Patientenorientierung .................................................... 178

5.2.3 Qualitätsmanagement................................................... 184

5.2.4 Neue Medien................................................................... 188

5.2.5 Public Health ................................................................ 194

5.3 Schlußfolgerungen ................................................................. 198

6 Beschäftigungsentwicklung und Wachstumsperspektiven im Gesundheitssystem ................................................................. 203

6.1 Gesundheit als Sektor der Dienstleistungsgesellschaft................... 203

6.2 Bedarfsprofile und Beschäftigungsentwicklung im Gesundheitswesen ................................................................. 207

6.2.1 Pflege und Gesundheitsversorgung im Alter...................... 211

6.2.2 Prävention und Rehabilitation............................................. 216

6.2.3 Psychosoziale Gesundheit.................................................. 220

6.3 Gesundheit als wirtschaftlicher Wachstumssektor? ......................... 223

6.3.1 Ansichten und Erfahrungen in Deutschland......................... 227

6.3.2 Ansichten und Erfahrungen in den Niederlanden ................ 233

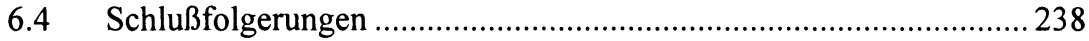

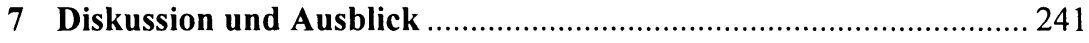

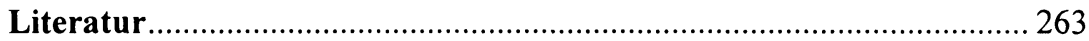

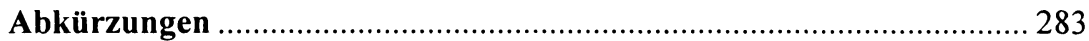




\section{Übersichten}

Abb. 1: Überblick über die Gliederung der Arbeit .............................. 16

Abb. 2: Akteur-Struktur-Dynamiken .................................................. 36

Abb. 3: Die Entwicklung wohlfahrtsstaatlicher Regimes:

Pfadabhängigkeit und Pfadwechsel in drei Phasen ................... 106

Abb. 4: Das Schalenmodell der Gesundheitswirtschaft ........................ 226

Tab. 1: Das gemischte Versicherungssystem der BRD und der Niederlande

Tab. 2: Vergütungssysteme in den Niederlanden und der Bundesrepublik

Tab. 3: Anteil der Gesundheitsausgaben in \% des BSP, Anteil einzelner Gesundheitsbereiche in \% an den Gesamtausgaben, Wachstum in \% 1980-96 85

Tab. 4: Ressourcen im Gesundheitswesen - Personal und Betten pro 1.000 Einwohner, Wachstum in \% 1980-96 .......................... 86

Tab. 5: Inanspruchnahme von Gesundheitsdiensten ........................... 87

Tab. 6: Wartelisten: Anzahl der Personen, die... ................................... 88

Tab. 7: Mortalitäts- und Morbiditätsindikatoren ................................ 89

Tab. 8: Indikatoren von Wohlfahrtsstaaten ....................................... 96

Tab. 9: Klassifikation von Industrieländern in Wohlfahrtsstaatstypen ... 99

Tab. 10: Die Einführung der sozialen Sicherungssysteme in

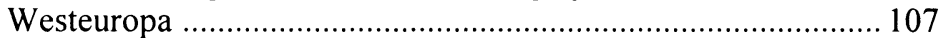

Tab. 11: Sozialleistungsquoten in Westeuropa .................................... 116

Tab. 12: Sektoren der Wohlfahrtsproduktion ...................................... 169

Tab. 13: Beschäftigungsentwicklung nach Sektoren in den Niederlanden .............................................................. 208

Tab. 14: Beschäftigungsentwicklung nach Sektoren in NRW .............. 208 
Tab. 15: Beschäftigungsentwicklung nach Berufen in den Niederlanden

Tab. 16: Beschäftigungsentwicklung nach Berufen in der BRD 210

Tab. 17: Ambulante und stationäre Kapazitäten der Wohlfahrtsverbände in der BRD

Tab. 18: Beschäftigungsentwicklung im Bereich Pflege und Versorgung in den Niederlanden .......................................................... 214

Tab. 19: Beschäftigungsentwicklung im Bereich Home Care ................ 215

Tab. 20: Beschäftigungsentwicklung in den Health Centres ................... 218

Tab. 21: Beschäftigungsentwicklung in Vorsorge- und RehaEinrichtungen in NRW

Tab. 22: Beschäftigungsentwicklung in der Prävention in den Niederlanden

Tab. 23: Beschäftigung in der ambulanten geistigen Gesundheitsversorgung (AGGZ) 222

Tab. 24: Beschäftigung in den Randsektoren des Gesundheitswesens in NRW 
„The first theorem goes like this: Regardless of the amount of money a nation spends on health care and regardless of the structure of its health system, people will complain about that health system. The second theorem is: In any country at any time there will always be a health reform. The third theorem is: In any country at any time people will say that the last health reform has been a failure. The fourth theorem is: As a Gesundheitssystemforscher, my husband will never be unemployed. And the fifth theorem is (...): Ministers of Health in any country must have done something bad in an earlier life. That is why they are Ministers of Health in that life. These are the theorems that in principle should be proven but they need no proof because my wife is never wrong."

Uwe E. Reinhardt in der Festrede zum Carl Bertelsmann-Preis 2000 